\title{
Intramyocardial hemorrhage and microvascular obstruction after primary percutaneous coronary intervention
}

\author{
A. M. Beek $\cdot$ R. Nijveldt $\cdot$ A. C. van Rossum
}

Received: 29 May 2009/ Accepted: 18 August 2009/Published online: 15 September 2009

(C) The Author(s) 2009. This article is published with open access at Springerlink.com

\begin{abstract}
Reperfusion may cause intramyocardial hemorrhage (IMH) by extravasation of erythrocytes through severely damaged endothelial walls. The purpose of the study was to evaluate the clinical significance of IMH in relation to infarct size, microvascular obstruction (MVO) and function in patients after primary percutaneous intervention. Forty-five patients underwent cardiovascular MR imaging (CMR) 1 week and 4 months after primary stenting for a first acute myocardial infarction. T2-weighted spin-echo imaging (T2W) was used to assess infarct related edema and $\mathrm{IMH}$, and delayed enhancement (DE) was used to assess infarct size and MVO. Cine CMR was used to assess left ventricular volumes and function at baseline and at 4 months follow-up. In $22(49 \%)$ patients, IMH was detected as areas of attenuated signal in the core of the high signal intensity region on T2W images. Patients with IMH had larger infarcts, higher left ventricular volumes and lower ejection fraction. Contrast-to-noise ratio (CNR) between hyperintense periphery and the hypo-intense core of the $\mathrm{T} 2 \mathrm{~W}$ ischemic area correlated to peak CKMB, total infarct size and MVO size. Using
\end{abstract}

A. M. Beek $(\varangle)$ · R. Nijveldt · A. C. van Rossum Department of Cardiology, VU University Medical Center, De Boelelaan 1117, 1081 HV Amsterdam, The Netherlands

e-mail: am.beek@vumc.nl

R. Nijveldt

Interuniversity Cardiology Institute of the Netherlands, Utrecht, The Netherlands univariable analysis, CNR predicted ejection fraction at baseline $(\beta=-0.62, P=0.003)$ and follow-up $(\beta=-0.84, P<0.001)$. However, after multivariable analysis, baseline ejection fraction and presence of MVO were the only parameters that predicted functional changes at follow-up. IMH was found in the majority of patients with MVO after reperfused myocardial infarction. It was closely related to markers of infarct size, MVO and function, but did not have prognostic significance beyond MVO.

Keywords Myocardial infarction . Myocardial reperfusion injury - Hemorrhage · Magnetic resonance imaging

\section{Introduction}

Timely reperfusion is the only way to preserve ischemic myocardium in acute myocardial infarction. However, reperfusion also induces new pathological changes that were not seen in the pre-interventional era [1]. Microvascular obstruction (MVO) or noreflow refers to the small vessel changes that prevent adequate tissue perfusion despite a revascularized and patent epicardial coronary artery [2, 3]. Although MVO is related to infarct size, it is an independent and powerful predictor of adverse outcome [4, 5]. Reperfusion may also cause intramyocardial hemorrhage (IMH) by extravasation of erythrocytes through 
severely damaged endothelial walls $[6,7]$. IMH can be visualized by T2-weighted CMR because breakdown products of hemoglobin are paramagnetic and influence regional magnetic tissue properties [8, 9]. Previous studies found IMH in about one-third of patients and suggested a relation with more severe myocardial injury and MVO [10, 11]. However, the exact significance of IMH and its relation to MVO remain unclear.

This study was undertaken to further evaluate presence and clinical significance of IMH by exploring its relation to infarct size, MVO and function in patients after primary coronary angioplasty for acute myocardial infarction.

\section{Methods}

Patients

All patients gave informed consent to the study protocol, which was approved by the local ethics committee. Patients were considered study candidates when admitted with a first ST-elevation acute myocardial infarction treated with successful primary coronary angioplasty (PCI) with stent implantation, defined as thrombolysis in myocardial infarction (TIMI) flow grade 2 or 3 . Patients with hemodynamic instability or (relative) contraindications for CMR were excluded.

Cardiac magnetic resonance imaging protocol

All examinations were performed on a 1.5-T clinical scanner (Sonata/Symphony, Siemens, Erlangen, Germany) using a phased array cardiac receiver coil. The baseline scan was scheduled between 2 and 9 days after reperfusion and follow-up at 4 months. ECGgated images were acquired during end-expiration breath holding. Segmented steady state free precession cine imaging (average voxel size $1.6 \times 1.9 \times 5 \mathrm{~mm}^{3}$, temporal resolution $47 \mathrm{~ms}$ ) was used to evaluate left ventricular function in long axis views and full coverage short axis views (interslice gap $5 \mathrm{~mm}$ ). Prior to contrast injection, breath-hold, segmented T2weighted spin-echo imaging (T2W) $(S T I R, \mathrm{TR}=$ $2 \times$ RR-interval, TE $64 \mathrm{ms,}$ average voxel size $1.4 \times 1.9 \times 7 \mathrm{~mm}^{3}$ ) was performed to visualize infarct related edema and hemorrhage. Three long axis views and a short axis view at the core of the infarct, defined as the short axis slice position with the largest circumferential amount of wall motion abnormalities were acquired [12]. An image normalisation filter was used to correct for coil inhomogeneity. Delayed enhancement (DE) images were acquired using a segmented inversion recovery gradient-echo pulse sequence (average voxel size $1.4 \times 1.7 \times$ $5 \mathrm{~mm}^{3}$, trigger pulse 2 , inversion time $250-300 \mathrm{~ms}$ ) 12-15 min after i.v. administration of $0.2 \mathrm{mmol} / \mathrm{kg}$ gadolinium-DTPA in short axis views with slice positions copied from the cine series.

\section{Analysis}

All CMR data were analysed on a separate workstation using dedicated software (Mass version 2006beta, Medis, Leiden, the Netherlands). Cine, T2W and contrast images were analysed separately, and, during analysis of one technique, the observers were blinded to results of the other techniques as well as to other clinical patient data. Endocardial and epicardial contours were manually drawn on all end-diastolic and end-systolic cine images to calculate normalised (body surface area) left ventricular end-diastolic (EDV) and end-systolic volumes (ESV), mass and ejection fraction. T2W images were assessed qualitatively for the presence of regional high signal intensity. IMH was considered present on T2W if regions of low (attenuated) signal were seen within the high signal area. Using the short axis view, the high signal intensity area (T2W infarct area) was quantified after windowing the images at mean +2 SD of the signal intensity (SI) of remote, normal myocardium, and expressed as a percentage of total slice area. IMH was included in the calculation of $\mathrm{T} 2 \mathrm{~W}$ infarct area. An ellipsoid region of interest $\left(0.2-0.3 \mathrm{~cm}^{2}\right)$ was placed in the central part (core) of the $\mathrm{T} 2 \mathrm{~W}$ infarct, covering the region with attenuated signal when present, and placed in the middle of the hyperintense region in patients without IMH. Regions of interest were also drawn in the peripheral part (peri) of the T2W infarct area and in remote, normal myocardium (Fig. 1). Signal-to-noise ratio (SNR) was calculated as $\mathrm{SNR}_{\text {core }}=\mathrm{SI}_{\text {core }}$ l $\mathrm{SD}_{\text {noise }}$, and $\mathrm{SNR}_{\text {peri }}=\mathrm{SI}_{\text {peri }} / \mathrm{SD}_{\text {noise }}$ and $\mathrm{SNR}_{\text {norm }}=$ $\mathrm{SI}_{\text {norm }} / \mathrm{SD}_{\text {noise }}$, respectively. Since no parallel imaging was used, noise was calculated as the standard deviation of a region of interest in background air, divided by 0.7 , taking into account the number of 
Fig. 1 Edema,

hemorrhage, infarct and microvascular obstruction. High and homogeneous $\mathrm{T} 2 \mathrm{~W}$ signal (a) in a patient with subendocardial anteroseptal infarction without MVO (b). Attenuated T2W signal corresponding to hemorrhage in the infarct core (c) in a patient with transmural inferoposterior infarction with MVO (d). Infarct area on $\mathrm{T} 2 \mathrm{~W}$ images is larger than on DE images in both patients. Borders of the infarcted areas are indicated by triangles, $\mathrm{T} 2 \mathrm{~W}$ core by solid arrow, T2W periphery by interrupted arrow and MVO by asterisk


effective radiofrequency coils.[13] Contrast-to-noise ratio (CNR) between the infarct periphery and core was calculated as $\mathrm{CNR}=\left(\mathrm{SI}_{\text {peri }}-\mathrm{SI}_{\text {core }}\right) / \mathrm{SD}_{\text {noise }}$. $\mathrm{DE}$ images were analysed as previously described [14]. Total infarct size was expressed as percentage of total left ventricular mass. MVO was defined as any region of hypoenhancement within the hyperenhanced, infarcted area, and was included in the calculation of total infarct size. MVO size was calculated by subtraction of the hyperenhanced area from the total infarct size, and expressed as a percentage of left ventricular mass (\%). For direct comparison of infarct extent with $\mathrm{T} 2 \mathrm{~W}$ images, infarct area in the same slice position was quantified and expressed as percentage of total slice area.

\section{Statistical analysis}

Data are expressed as mean $\pm \mathrm{SD}$ for continuous variables. The independent samples $t$ test and Chisquare test were used to compare subgroups. The paired samples $t$ test was used to evaluate changes in left ventricular global parameters between baseline and follow-up. Pearson's correlation coefficients (r) were calculated for the relation between $\mathrm{CNR}$, peak $\mathrm{CKMB}$, total infarct size, MVO size and ejection fraction. Univariable and multivariable linear regression analysis was used to evaluate the relation of clinical (age, CKMB) and CMR parameters (total infarct size, MVO, CNR) to ejection fraction and their ability to predict changes at follow-up.

\section{Results}

Fifty consecutive patients were included in the study protocol. T2W image quality was non-diagnostic in both short axis and long axis views in $5(10 \%)$ because of artifacts or regional signal loss caused by cardiac or respiratory motion. The remaining 45 patients had an uncomplicated clinical course between both CMR examinations. Mean time between admission and baseline CMR was $5.1 \pm 2.1$ days. Mean total infarct size was $17.6 \pm 10.0 \%$ of total left ventricular mass. 
MVO was identified on contrast images in 27 patients $(60 \%)$, and mean MVO size was $6.0 \pm 8.4 \%$ of total infarct size. Mean ejection fraction was $42 \pm 10 \%$, and increased to $45 \pm 10 \%$ at follow-up $(P=0.004)$.

Edema, hemorrhage and MVO

In all patients, T2W revealed a region of high signal intensity in the distribution area of the infarct related artery (Fig. 1). In almost half (22 of 45), areas of varying size of lower signal intensity could be identified in the central part of the area of high signal intensity (Fig. 1). Baseline characteristics according to presence of IMH are summarized in Table 1.

All patients with IMH had MVO on contrast images and only five patients with MVO did not have IMH on T2W. At baseline, patients with IMH had higher peak CKMB, larger total infarct size, larger volumes and lower ejection fraction. At follow-up, patients without IMH showed a significant improvement in EDV $\left(89 \pm 22\right.$ vs. $83 \pm 22 \mathrm{ml} / \mathrm{m}^{2}, P=$ $0.05), \mathrm{ESV}\left(49 \pm 17\right.$ vs. $\left.43 \pm 15 \mathrm{ml} / \mathrm{m}^{2}, P=0.003\right)$ and ejection fraction $(46.4 \pm 8.4$ vs. $49.6 \pm 8.0 \%$, $P=0.04)$. Patients with IMH showed a non-significant increase in EDV $(104 \pm 22$ vs. $111 \pm 29 \mathrm{ml} /$ $\left.\mathrm{m}^{2}, P=0.076\right)$ and ESV $(65 \pm 19$ vs. $67 \pm 24 \mathrm{ml} /$ $\mathrm{m}^{2}, P=0.310$ ), and a small, non-significant increase in ejection fraction $(38.9 \pm 8.1$ vs. $40.6 \pm 8.5 \%$, $P=0.72)$.

\section{SNR and CNR analysis}

Five additional patients were excluded from further signal intensity analysis because the short axis images were of insufficient quality to calculate $\mathrm{T} 2 \mathrm{~W}$ infarct area or SNR. In the remaining 40 patients, there was a significant correlation between mean infarct area on T2W images and on DE images $(\mathrm{r}=0.73, P<$ 0.001 ), although the area was larger on $\mathrm{T} 2 \mathrm{~W}$ images: $49.3 \pm 15 \%$ vs. $28.9 \pm 15.7 \%, P<0.001$ (Fig 1 ). $\mathrm{SNR}_{\text {peri }}$ was significantly higher than $\mathrm{SNR}_{\text {core }}$ in the patients with MVO (29.4 vs. 20.6, $P<0.001)$ but not in patients without MVO (29.9 vs. $30.2, P=0.68)$. As a result, CNR was higher in patients with MVO than in patients without MVO $(8.8 \pm 5.5$ vs. $0.3 \pm$ $3.5, P<0.001)$. CNR correlated significantly with peak CKMB (r $=0.51, P=0.001)$, total infarct size
Table 1 Baseline characteristics according to absence (IMH-) or presence $(\mathrm{IMH}+)$ of intramyocardial hemorrhage on $\mathrm{T} 2 \mathrm{~W}$ images

\begin{tabular}{|c|c|c|c|}
\hline & IMH- & $\mathrm{IMH}+$ & $P$ value \\
\hline Number & 23 & 22 & NS \\
\hline Age (SD) & $59 \pm 10$ & $54 \pm 10$ & NS \\
\hline Male & 21 & 19 & NS \\
\hline Diabetes & 2 & 0 & NS \\
\hline Hypercholesterolemia & 5 & 8 & NS \\
\hline Hypertension & 6 & 6 & NS \\
\hline Smoking & 14 & 14 & NS \\
\hline \multicolumn{4}{|l|}{ Infarct related artery } \\
\hline LAD & 13 & 16 & NS \\
\hline $\mathrm{LCx}$ & 3 & 3 & NS \\
\hline RCA & 7 & 3 & NS \\
\hline Time to reperfusion (hours) & $3.6 \pm 3.8$ & $3.5 \pm 3.0$ & NS \\
\hline Abciximab & 19 & 20 & NS \\
\hline \multicolumn{4}{|l|}{ TIMI flow post-PCI } \\
\hline TIMI 2 & 4 & 3 & NS \\
\hline TIMI 3 & 19 & 19 & NS \\
\hline Peak CKMB & $188 \pm 102$ & $378 \pm 156$ & $<0.001$ \\
\hline $\begin{array}{l}\text { Time to baseline } \\
\text { CMR (days) }\end{array}$ & $4.3 \pm 2.1$ & $5.7 \pm 2.0$ & 0.03 \\
\hline Total infarct size (\% of LV) & $11.3 \pm 7.3$ & $24.4 \pm 7.0$ & $<0.001$ \\
\hline MVO & 5 & 22 & $<0.001$ \\
\hline MVO (\% of total infarct) & $0.3 \pm 1.1$ & $12.3 \pm 8.9$ & $<0.001$ \\
\hline $\operatorname{EDV}\left(\mathrm{ml} / \mathrm{m}^{2}\right)$ & $89 \pm 22$ & $104 \pm 22$ & 0.02 \\
\hline $\operatorname{ESV}\left(\mathrm{ml} / \mathrm{m}^{2}\right)$ & $49 \pm 17$ & $64 \pm 19$ & 0.007 \\
\hline Ejection fraction $(\%)$ & $46.4 \pm 8.4$ & $38.9 \pm 8.1$ & 0.004 \\
\hline
\end{tabular}

Values are presented as numbers or as mean \pm standard deviation $L A D$ left anterior descending artery, $L C x$ left circumflex artery, $R C A$ right coronary artery, TIMI thrombolysis in myocardial infarction. $P C I$ percutaneous coronary intervention

( $\mathrm{r}=0.57, P<0.001)$, and size of the MVO area $(\mathrm{r}=0.52, P<0.001)$.

Hemorrhage and ejection fraction

Using univariable analysis, CNR strongly predicted ejection fraction at baseline $(\beta=-0.62, P=0.003)$ and follow-up $(\beta=-0.84, P<0.001)$, but not the change over time $(\beta=-0.22, P=0.11)$. After multivariable analysis, infarct size remained the single independent predictor of ejection fraction at baseline $(\beta=-0.69, P<0.001)$ and follow-up $(\beta=-0.73$, $P<0.001)$, whereas baseline ejection fraction and presence of MVO were the only predictors of change 
in ejection fraction $(\beta=-0.29$ and $\beta=-4.99$, respectively, $P<0.01)$.

\section{Discussion}

Our study showed that intramyocardial hemorrhage can be found in almost half of patients with successfully revascularized acute myocardial infarction. The presence of IMH was associated with larger infarcts, presence of MVO, higher left ventricular volumes and lower ejection fraction, and the lack of improvement at follow-up. Contrast-to-noise ratio between (attenuated) core and (high) peripheral T2W signal intensity was strongly related to markers of infarct size, MVO and function. However, in our study, it was not an independent predictor of functional changes at follow-up.

\section{T2-weighted imaging, edema and hemorrhage}

Normal myocardium has low to intermediate signal on T2W images, but increased regional water content, such as infarct related edema, causes an increase in the T2-relaxation time and T2W signal intensity [15]. Hemorrhage and the breakdown of oxygenated hemoglobin also influence magnetic properties of the surrounding tissue. The effects of hemoglobin degradation have been extensively studied in patients with cerebral hemorrhage; they are complex and strongly dependent on the age of the hematoma and the integrity of the erythrocyte membrane [8]. T2W signal is high in the very early, hyperacute phase, but then falls because of the paramagnetic effects of deoxyhemoglobin and intracellular methemoglobin [8]. Especially in the core of the hematoma, where there is marked hypoxia, signal may remain very low for a prolonged period of time [16]. Lotan et al. studied IMH in a canine myocardial infarction model using ex-vivo T2W [9]. They found areas of low signal within the zone of increased signal that accurately matched the location of macroscopic hemorrhage in all animals but one (with a very small hemorrhage), and no hypointense $\mathrm{T} 2 \mathrm{~W}$ regions in the animals without hemorrhage. Hemorrhage size according to CMR correlated closely to size determined from tissue slices and according to labeled red blood cells. Basso et al. recently compared in vivo and ex-vivo CMR to histopathological findings in two patients that had died 12 and 24 days after percutaneous revascularization, respectively [17]. Both patients had hemorrhagic infarcts, with massive bleeding at the core of the infarct. Both in vivo and ex-vivo T2W showed areas of low signal intensity within areas of high signal intensity that corresponded to hemorrhage and edema, respectively. Thus, although we have no direct proof of the presence of hemorrhage in our patients, we believe that these studies sufficiently show that hemorrhage and hemoglobin breakdown leads to T2W signal attenuation as documented in our study. Our results are roughly in line with the results from previous studies that used $\mathrm{T} 2 *$ ('star')-weighted gradient-echo techniques to visualize IMH [10, 11]. Asanuma et al. found IMH in 9 of 24 (38\%) patients with reperfused anterior MI [10]. Patients with IMH had larger enzymatic infarct size, more Q-wave infarctions and less improvement in echocardiographic wall motion score. Ochiai et al. showed IMH in 13 of 39 (33\%) patients with reperfused infarction, and also found an association between IMH, infarct severity and less improvement of ventriculographic ejection fraction [11]. There are no studies comparing $\mathrm{T} 2 \mathrm{~W}$ spinecho imaging to $\mathrm{T} 2 *$ weighted gradient-echo imaging for the assessment of $\mathrm{IMH}$, and, at this point, it is not clear which is the optimal technique for its visualisation. Cardiac application of both techniques has been validated in a limited number of experimental and pathological studies $[9,11,18]$. Although T2*-weighted gradientecho imaging is very sensitive to the paramagnetic effects of the deoxyhemoglobin and methemoglobin, it requires relatively long echo times that may degrade image quality during cardiac imaging [8]. T2W spinecho imaging has the advantage that it also depicts infarct related edema, which has been shown to correspond to the area at risk [19, 20]. In line with a recent report by Friedrich et al., we found that mean (single slice) T2W infarct area was (approximately $70 \%)$ larger than DE infarct area [20].

\section{Clinical implications}

Hemorrhagic myocardial infarction was rarely seen in autopsy studies in the pre-reperfusion era, but its reported incidence markedly increased after the introduction of thrombolytic therapy [6, 21]. Later studies showed that it also occurred after primary PCI $[10,11]$. The appearance of hemorrhage therefore 

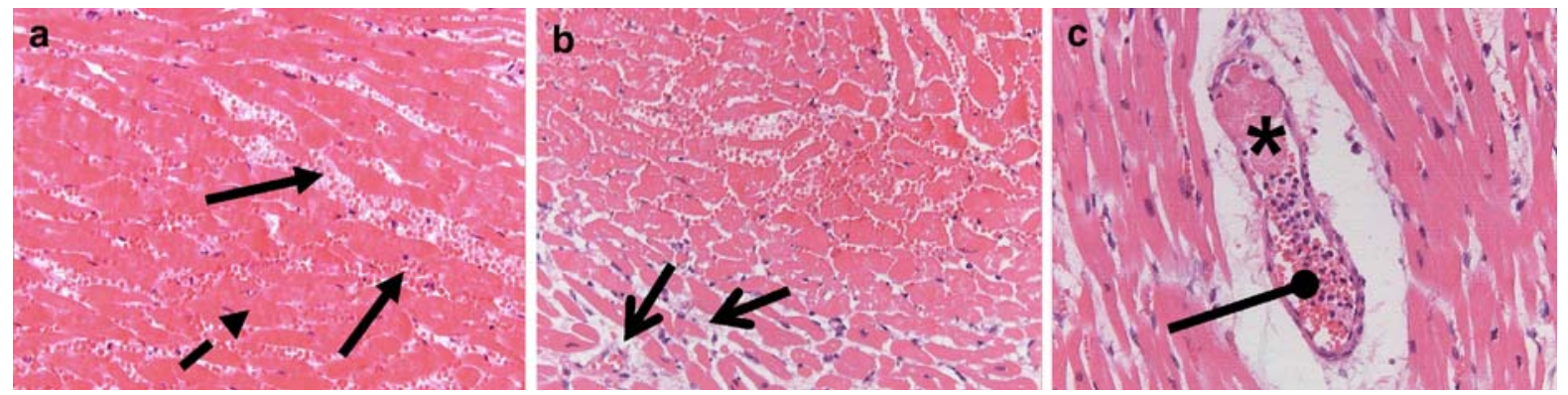

Fig. 2 Microscopic slides at $200 \times$ magnification after standard hematoxylin and eosin staining of three samples of the heart of a 44-year-old man who died 3 days after admission with acute inferoposterior infarction (patient not included in the study group). (a) infarct core with necrotic cardiomyocytes

depends on the coexistence of myocardial reperfusion and microvascular damage in the setting of severe myocardial ischemia $[7,17]$. The large majority $(81 \%)$ of our patients with MVO also had IMH, and MVO size in the 5 patients without IMH was very small $(<1 \%$ of total infarct size). Further more, our results suggest that hemorrhage severity, as reflected by the degree of $\mathrm{T} 2 \mathrm{~W}$ signal attenuation as contrast-to-noise ratio, is related to infarct and MVO extent, but does not have prognostic value beyond MVO. In early studies in the mid-seventies, Kloner et al. already demonstrated that extravasation of erythrocytes is one of the morphological features found in areas of no-reflow [22]. This close relation between MVO and IMH might explain the lack of additional prognostic significance as seen in our study. Figure 2 illustrates the co-existence of edema, IMH and MVO in a patient who died 3 days after inferoposterior myocardial infarction (patient was not included in the study group).

The non-invasive assessment of IMH by CMR might be used to indicate infarct severity and underlying microvascular injury, e.g. in patients with (moderate to severe) renal insufficiency, who have a relative contra-indication against the use of gadolinium compounds [23]. However, further study is needed to define the optimum CMR technique to assess IMH, and to determine the exact role of IMH in the myocardial healing process.

\section{Study limitations}

Spatial coverage of the $\mathrm{T} 2 \mathrm{~W}$ images was limited as we focused on the identification of hemorrhage in the core of the infarct. As a result, we may have missed (interrupted arrow) and abundant erythrocytes (solid arrows), (b) infarct border zone with edema (open arrow), (c) capillary vessel with thrombus (asterisk) and plugged polymorphonuclear cells (circled arrow)

regions of signal attenuation in adjacent slices in the five patients with MVO but without IMH.

Signal-to-noise ratios are related to scan parameters such as voxel size, and therefore our results may not be directly applicable when other scanners are used.

The current breath-hold T2W spin-echo imaging technique is limited by its sensitivity to artifacts caused by cardiac or respiratory motion, as illustrated by the considerable number of non-diagnostic images in our study group (10\% of long axis images, $20 \%$ of short axis images). A recently proposed adaptation of the technique to optimize performance has not yet been evaluated in a clinical setting [24].

\section{Conclusions}

Intramyocardial hemorrhage was found in the majority of patients with microvascular obstruction after percutaneous revascularization for acute myocardial infarction. It was closely related to markers of infarct size, MVO and function, but, in this study, did not have prognostic significance beyond MVO.

Acknowledgements The authors would like to thank Paul Algra, MD, PhD (department of Radiology, Medical Center, Alkmaar, The Netherlands), Victor Umans, $\mathrm{MD}, \mathrm{PhD}$ (department of Cardiology Medical Center, Alkmaar, The Netherlands) and Mary Belderok (department of Cardiology, VU University Medical Center, Amsterdam) for their contribution to the patient group collection and the data acquisition. The authors would also like to thank Hans Niessen, MD, PhD (department of Pathology, VU University Medical Center, Amsterdam, The Netherlands) for providing Fig. 2. This study was supported by Netherlands Heart Foundation grant 2003B126. 
Ethical standards This study was approved by the local ethics committee. Written informed consentwas obtained from all patients.

Open Access This article is distributed under the terms of the Creative Commons Attribution Noncommercial License which permits any noncommercial use, distribution, and reproduction in any medium, provided the original author(s) and source are credited.

\section{References}

1. Pasotti M, Prati F, Arbustini E (2006) The pathology of myocardial infarction in the pre- and post-interventional era. Heart 92:1552-1556

2. Ambrosio G, Weisman HF, Mannisi JA et al (1989) Progressive impairment of regional myocardial perfusion after initial restoration of postischemic blood flow. Circulation 80:1846-1861

3. Reffelmann T, Kloner RA (2002) The "no-reflow" phenomenon: basic science and clinical correlates. Heart $87: 162-168$

4. Wu KC, Zerhouni EA, Judd RM et al (1998) Prognostic significance of microvascular obstruction by magnetic resonance imaging in patients with acute myocardial infarction. Circulation 97:765-772

5. Nijveldt R, Beek AM, Hirsch A et al (2008) Functional recovery after acute myocardial infarction: a comparison between angiography, electrocardiography and cardiovascular magnetic resonance measures of microvascular injury. J Am Coll Cardiol 52:181-189

6. Fujiwara H, Onodera T, Tanaka M et al (1986) A clinicopathologic study of patients with hemorrhagic myocardial infarction treated with selective coronary thrombolysis with urokinase. Circulation 73:749-757

7. Basso C, Thiene G (2006) The pathophysiology of myocardial reperfusion: a pathologist's perspective. Heart 92:1559-1562

8. Bradley WG Jr (1993) MR appearance of hemorrhage in the brain. Radiology 189(1):15-26

9. Lotan CS, Bouchard A, Cranney GB et al (1992) Assessment of postreperfusion myocardial hemorrhage using proton NMR imaging at $1.5 \mathrm{~T}$. Circulation 86:1018-1025

10. Asanuma T, Tanabe K, Ochiai K et al (1997) Relationship between progressive microvascular damage and intramyocardial hemorrhage in patients with reperfused anterior myocardial infarction: myocardial contrast echocardiographic study. Circulation 96:448-453

11. Ochiai K, Shimada T, Murakami Y et al (1999) Hemorrhagic myocardial infarction after coronary reperfusion detected in vivo by magnetic resonance imaging in humans: prevalence and clinical implications. J Cardiovasc Magn Reson 1:247-256

12. Simonetti OP, Finn JP, White RD et al (1996) "Black blood" T2-weighted inversion-recovery MR imaging of the heart. Radiology 199:49-57

13. Constantinides CD, Ergin A, McVeigh E (1997) Signal-tonoise measurements in magnitude images from NMR phased arrays. Magn Reson Med 38:852-857

14. Nijveldt R, Beek AM, Hofman MBM et al (2007) Late gadolinium-enhanced cardiovascular magnetic resonance evaluation of infarct size and microvascular obstruction in optimally treated patients after acute myocardial infarction. J Cardiovasc Magn Reson 9:765-770

15. Higgins CB, Herfkens R, Lipton MJ et al (1983) Nuclear magnetic resonance imaging of acute myocardial infarction in dogs: alterations in magnetic relaxation times. Am J Cardiol 52:184-188

16. Parizel PM, Makkat S, Van Miert E et al (2001) Intracranial hemorrhage: principles of CT and MRI interpretation. Eur Radiol 11:1770-1783

17. Basso C, Corbetti F, Silva C et al (2007) Morphologic validation of reperfused hemorrhagic myocardial infarction by cardiovascular magnetic resonance. Am J Cardiol 100:1322-1327

18. van den Bos EJ, Baks T, Moelker AD et al (2006) Magnetic resonance imaging of hemorrhage within reperfused myocardial infarcts: possible interference with iron oxidelabelled cell tracking? Eur Heart J 27:1620-1626

19. Aletras AH, Tilak GS, Natanzon A et al (2006) Retrospective determination of the area at risk for reperfused acute myocardial infarction with T2-weighted cardiac magnetic resonance imaging: histopathological and displacement encoding with stimulated echoes (DENSE) functional validations. Circulation 113:1865-1870

20. Friedrich MG, Abdel-Aty H, Taylor A et al (2008) The salvaged area at risk in reperfused acute myocardial infarction as visualized by cardiovascular magnetic resonance. J Am Coll Cardiol 51:1581-1587

21. Mathey DG, Schofer J, Kuck KH et al (1982) Transmural, hemorrhagic myocardial infarction after intracoronary streptokinase. Clinical, angiographic, and necropsy findings. Br Heart J 48(6):546-551

22. Kloner RA, Ganote CE, Jennins RB et al (1975) Demonstration of the "no-reflow" phenomenon in the dog heart after temporary ischemia. Recent Adv Stud Cardiac Struct Metab 10:463-474

23. Kuo PH, Kanal E, Abu-Alfa AK et al (2007) Gadoliniumbased MR contrast agents and nephrogenic systemic fibrosis. Radiology 242:647-649

24. Keegan J, Gatehouse PD, Prasad SK et al (2006) Improved turbo spin-echo imaging of the heart with motion-tracking. J Magn Reson Imaging 24:563-570 